\title{
Simultaneous Recordings from the Primary Visual Cortex and Lateral Geniculate Nucleus Reveal Rhythmic Interactions and a Cortical Source for Gamma-Band Oscillations
}

\author{
Andre M. Bastos, ${ }^{1,2,5}$ Farran Briggs, ${ }^{1,6}$ Henry J. Alitto, ${ }^{1,3}$ George R. Mangun, ${ }^{2,4,7}$ and W. Martin Usrey ${ }^{1,3,7}$ \\ Centers for ${ }^{1}$ Neuroscience, ${ }^{2}$ Mind and Brain, Departments of ${ }^{3}$ Neurobiology, Physiology and Behavior, and ${ }^{4}$ Psychology, University of California Davis, \\ Davis, California 95616, ${ }^{5}$ Ernst Strüngmann Institute for Neuroscience in Cooperation with Max Planck Society, 60528 Frankfurt, Germany, ${ }^{6}$ Department of \\ Physiology and Neurobiology, Geisel School of Medicine at Dartmouth, Lebanon, New Hampshire 03756, and 7Department of Neurology, University of \\ California Davis, Sacramento, California 95817
}

Oscillatory synchronization of neuronal activity has been proposed as a mechanism to modulate effective connectivity between interacting neuronal populations. In the visual system, oscillations in the gamma-frequency range $(30-100 \mathrm{~Hz})$ are thought to subserve corticocortical communication. To test whether a similar mechanism might influence subcortical-cortical communication, we recorded local field potential activity from retinotopically aligned regions in the lateral geniculate nucleus (LGN) and primary visual cortex (V1) of alert macaque monkeys viewing stimuli known to produce strong cortical gamma-band oscillations. As predicted, we found robust gammaband power in V1. In contrast, visual stimulation did not evoke gamma-band activity in the LGN. Interestingly, an analysis of oscillatory phase synchronization of LGN and V1 activity identified synchronization in the alpha $(8-14 \mathrm{~Hz})$ and beta (15-30 Hz) frequency bands. Further analysis of directed connectivity revealed that alpha-band interactions mediated corticogeniculate feedback processing, whereas beta-band interactions mediated geniculocortical feedforward processing. These results demonstrate that although the LGN and V1 display functional interactions in the lower frequency bands, gamma-band activity in the alert monkey is largely an emergent property of cortex.

Key words: cortex; LGN; monkey; oscillations; V1; vision

\section{Introduction}

Understanding how visual information is dynamically routed across the complex network of visual brain areas is an important and unresolved question. Recent theories on the role of gammaband oscillations in visual processing emphasize a putative role in enhancing interareal interactions (Fries, 2005; Gregoriou et al., 2009). Along these lines, an emerging view is that gamma-band oscillations signal information in the feedforward or bottom-up direction of processing (Buschman and Miller, 2007; Bastos et al., 2012; Bosman et al., 2012; Roberts et al., 2013). Although gamma-band activity in precortical structures has been reported, the evidence in support of this in mammals comes

\footnotetext{
Received Oct. 1, 2013; revised March 31, 2014; accepted April 24, 2014.

Author contributions: A.M.B., F.B., G.R.M., and W.M.U. designed research; F.B. and H.J.A. performed research; A.M.B. and H.J.A. analyzed data; A.M.B., F.B., G.R.M., and W.M.U. wrote the paper.

Supported by NIH Grants EY013588 (W.M.U.), EY018683 (F.B.), and MH055714 (G.R.M.), and NSF Grants BCS0727115 and BCS-1228535 (G.R.M. and W.M.U.), and an NSF Graduate Research Fellowship 2009 (090358; A.M.B.). We thank K.E. Neverkovec, D.J. Sperka, and R. Oates-0'Brien for assistance, Pascal Fries for advice, and Michael Schmid for comments on the paper.

The authors declare no competing financial interests.

Correspondence should be addressed to Dr W. Martin Usrey, University of California Davis, 1544 Newton Court, Davis, CA 95618. E-mail: wmusrey@ucdavis.edu.

DOI:10.1523/JNEUROSCI.4216-13.2014

Copyright $\odot 2014$ the authors $\quad 0270-6474 / 14 / 347639-06 \$ 15.00 / 0$
}

from studies that made measurements in anesthetized cats (Neuenschwander and Singer, 1996; Castelo-Branco et al., 1998; Koepsell et al., 2009). Given the influence anesthesia can have on rhythmic brain activity (Xing et al., 2012a; Saxena et al., 2013; Vijayan et al., 2013), it is critical to investigate gamma-band activity and interareal interactions in the alert state.

We made simultaneous recordings from the lateral geniculate nucleus (LGN) and primary visual cortex (V1) in alert macaque monkeys to determine whether oscillatory interactions in the gamma-frequency band support feedforward subcortical-tocortical visual processing. Recordings were made while animals passively viewed drifting grating stimuli that have previously been shown to induce significant gamma activity in visual cortex (Bosman et al., 2012; Roberts et al., 2013). Despite finding robust visually evoked gamma-frequency activity in V1, we did not observe visually evoked gamma activity in the LGN. The LGN and V1, however, did display prominent oscillatory interactions, in a broad range of subgamma frequencies, with alpha-band frequencies reflecting corticogeniculate feedback interactions, and beta-band frequencies reflecting geniculocortical feedforward interactions. These results indicate that network gamma-band oscillations observed between visual cortical areas (Gregoriou et al., 2009; Bosman et al., 2012; 
Roberts et al., 2013) are an emergent feature of corticocortical interactions, but not geniculocortical interactions.

\section{Materials and Methods}

Four macaque monkeys (Macaca mulatta; two female and two male) were used in this study. All procedures were approved by the Institutional Animal Care and Use Committee at the University of California, Davis, and conformed to NIH guidelines. Surgical procedures were described previously (Briggs and Usrey, 2009). Briefly, under full surgical anesthesia, a head post, and two recording cylinders were secured to the skull. One recording cylinder was positioned above the LGN, and the other was positioned above V1.

Visual stimulation. Visual stimuli were generated with a VSG 2/5 (Cambridge Research Systems) and presented on a gamma-calibrated Sony monitor $(140 \mathrm{~Hz}$; mean luminance $38 \mathrm{~cd} /$ $\mathrm{m}^{2}$ ) located $700 \mathrm{~mm}$ in front of the animal. Animals were trained to fixate on a central dot for a liquid reward. Trials were aborted if eye position deviated by $>0.35^{\circ}$. Eye position was monitored with an infrared video eye tracker (Applied Science Laboratories; refresh rate 240 $\mathrm{Hz}$ ). Five hundred $\mathrm{ms}$ after obtaining fixation, a drifting sinusoidal grating was presented for $1.2-2.5 \mathrm{~s}$. Grating orientation and spatial frequency were set to match the overall preference of neurons at the V1 recording site. Stimulus contrast was $60-70 \%$ (except one session in which the contrast was $20 \%$ ), temporal frequency was $4 \mathrm{~Hz}$, and the diameter of the sinusoidal grating patch was $2^{\circ}$.

Electrophysiology. Twenty-one simultaneous recordings of local field potentials (LFPs) were made from the LGN and V1 in two monkeys and 209 single-unit recordings were made from the LGN in two additional monkeys. For the LFP recordings, single platinum/iridium electrodes $(\sim 0.1 \mathrm{M} \Omega$; FHC) were semichronically implanted within parafoveal regions of the LGN. V1 recordings were made with platinum-in-glass electrodes (0.5-1 M $\Omega$; Alpha Omega). Voltage recordings from LGN and V1 electrodes were amplified and recorded by a PC equipped with a Power 1401 data acquisition system and Spike2 software package (Cambridge Electronic Design). Neuronal activity was recorded at $20 \mathrm{kHz}$, with a $60 \mathrm{~Hz}$ hardware notch filter. Off-line, the signals were low-pass filtered at $120 \mathrm{~Hz}$ and down-sampled to $1000 \mathrm{~Hz}$ to extract LFPs. LGN and V1 electrodes were placed in precise retinotopic alignment (Briggs et al., 2013), such that receptive fields from the two locations were within $<2^{\circ}$ of one another in visual space (range: $0.2-1.8^{\circ}$, mean: $1.0^{\circ}$ ). Online assessment of receptive field overlap was confirmed by offline analysis of event-related potentials in the simultaneously recorded LGN and V1 channels.

Single-unit recordings were made from LGN neurons with platinumin-glass electrodes (Alpha Omega). Responses were amplified, filtered and recorded to a PC computer with a Power 1401 data acquisition interface and Spike 2 software (Cambridge Electronic Design).

Data analyses. Analyses of LFP signals were performed using the Fieldtrip (Oostenveld et al., 2011) open source MATLAB toolbox and custom MATLAB scripts (MathWorks). Data were normalized within each recording session so that the mean of each channel for each session was zero and the SD was one. Data were then pooled across sessions. Artifact rejection was performed using a semiautomatic procedure based on variance, maximum/minimum, and velocity criteria. Less than $10 \%$ of the trials were rejected. This yielded a total of 1669 (Monkey B) and 1184 (Monkey O) trials. For all nonaborted trials, LGN and V1 recordings

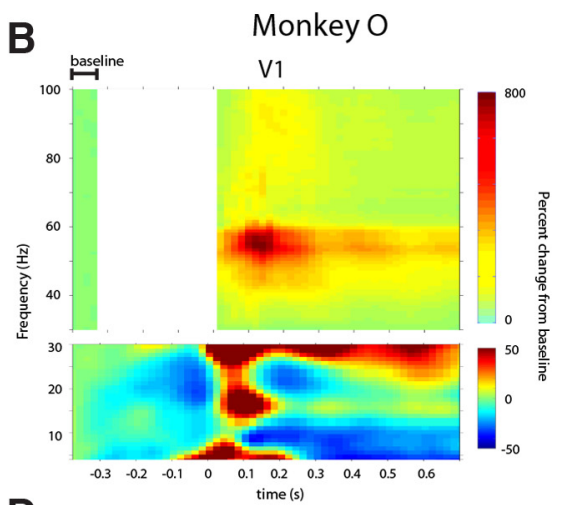

D

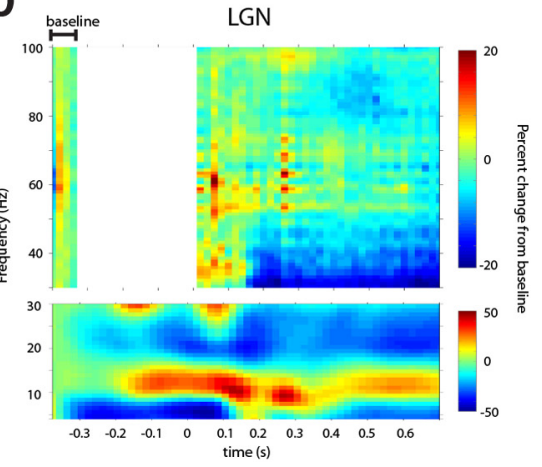



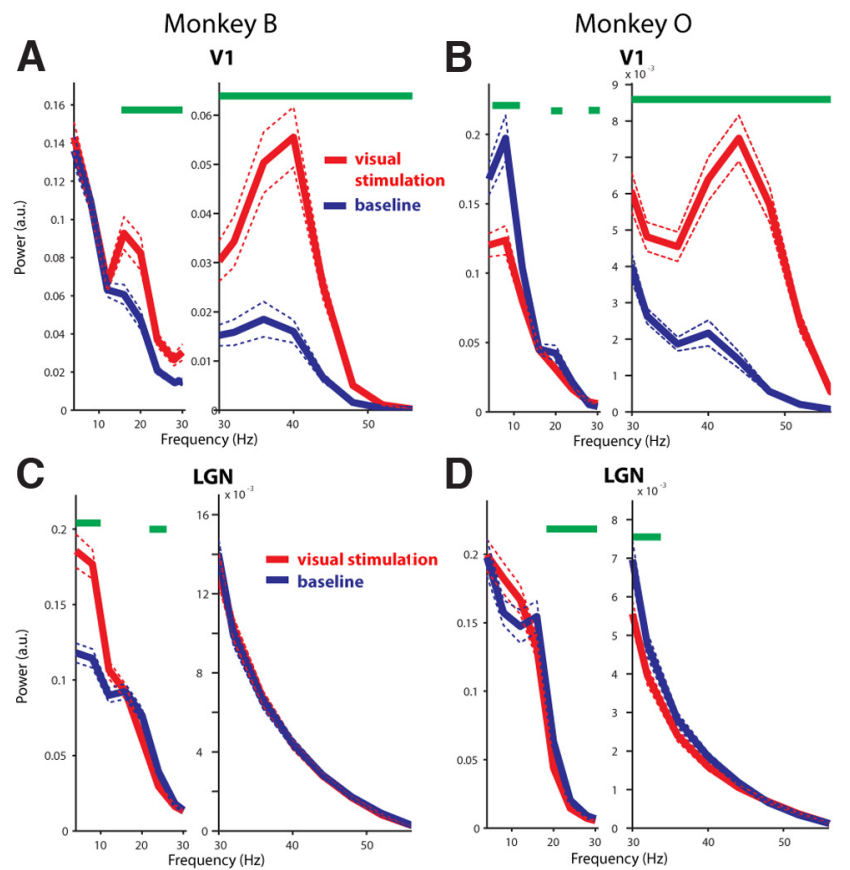

Figure 2. Power spectra of baseline and visual stimulation periods. $A, B$, Results from cortical recordings in Monkeys $B$ and 0 , respectively. Red traces show power during visual stimulation and blue traces show power during the prestimulation baseline in which the monkeys maintained fixation on a central dot. Dotted lines represent the mean \pm 2 SEM. Green bars indicate frequency bins that are significantly different between conditions. a.u., Arbitrary units. The spectra are broken between lower frequencies and frequencies surrounding the gamma peak to enhance visualization of the peaks. $\boldsymbol{C}, \boldsymbol{D}$, Results from LGN recordings in Monkeys $B$ and 0 , respectively. Conventions similar to $\boldsymbol{A}$ and $\boldsymbol{B}$.

frequency. This product is integrated over a series of frequency bins to produce an estimate of directed influence at a frequency $\mathrm{f}$. For this analysis, an integration window of $\pm 5 \mathrm{~Hz}$ was used, although small changes in the integration window did not qualitatively affect the results. When the PSI produces a positive value at a frequency, $f$, this indicates that V1 precedes LGN (a feedback influence) at that frequency, whereas if the value is negative, it indicates that the LGN precedes V1 (a feedforward influence).

Throughout, we have plotted the mean of the power, PPC, and PSI estimates \pm SEM (calculated using jackknife resampling). These estimates are provided to aid data visualization and to depict the statistical confidence associated with each estimate.

Statistical tests. We used nonparametric randomization testing to determine statistical significance of each data feature (stimulus vs baseline power contrast, non-zero PSI, and correlation across sessions between PPC and V1 power) and to correct for multiple comparisons across frequencies. This entails creating a reference distribution by randomizing a particular data feature to test the null hypothesis that, for example: (1) there is no difference between prestimulus power versus poststimulus onset power, (2) there is no significant leading/lagging relationship between the LGN and V1, and (3) there is no significant correlation over sessions between LGN-V1 PPC and V1 power. The reference distribution is created by performing the randomization 1000 times, and for each randomization, taking the maximum and minimum value of the respective data feature over frequencies. This procedure corrects for multiple comparisons over frequencies, and creates a reference distribution to which the empirical value is compared. If the empirical value is greater than the 97.5th percentile of the reference distribution (created from the maximum values over randomizations) or less than the 2.5 th percentile value of this reference distribution, (created from the minimum values over randomizations) it is considered statistically significant in a twosided test, corresponding to $p=0.05$.

\section{Results}

We made simultaneous recordings of visually evoked responses in the LGN and V1 of two alert macaque monkeys. In V1 of both animals, visual stimulation by the drifting sinusoidal grating increased gamma-band power in the frequency range of $\sim 30-100$ $\mathrm{Hz}$ (Fig. $1 A, B$, top). In contrast, visual stimulation did not significantly modulate power in the gamma frequency range in the LGN (Figs. $1 C, D$, top). Instead, power modulation in the LGN was concentrated at lower-frequencies, with visual stimulation decreasing beta-band power $(20-30 \mathrm{~Hz})$ and increasing alphaband power (centered at $10 \mathrm{~Hz}$; Fig. 1C,D, bottom). Within the LGN, the onset of low-frequency events begins slightly before the onset of visual stimulation, which may reflect activity changes associated with fixation and/or stimulus expectation.

To characterize the visually induced modulations of gamma-band activity further, we compared spectral power in the baseline period (500-250 ms before visual-stimulationonset) with a time window centered from 100 to $350 \mathrm{~ms}$ poststimulus onset. Figure 2 shows the respective power spectra for the LGN and V1. These spectra confirm the presence of an oscillatory gamma-band peak in the cortex $(\sim 30-55 \mathrm{~Hz})$ but not in the LGN. In the cortex, this peak increased significantly with visual stimulation (frequencies of increase: Monkey B, 16$100 \mathrm{~Hz}$; Monkey O, 28-100 Hz; $p<0.05$, randomization test; Fig. $2 A, B)$. Along these lines, the stimulus-to-baseline power ratio revealed that the gamma frequency that was maximally enhanced by visual stimulation was $44 \mathrm{~Hz}$ in Monkey $\mathrm{B}$, and $52 \mathrm{~Hz}$ in Monkey $\mathrm{O}$ (Fig. $3 A, B$ ). Although visual stimulation did not affect gamma power in the LGN ( $p>0.05$, randomization test), visual stimulation did modulate lower frequency power (Fig. $2 C, D$ ). In particular, alpha-band power was enhanced (frequencies of increase: $4-10 \mathrm{~Hz}$, Monkey B, $p<0.05$, nonsignificant but similar trend in Monkey $\mathrm{O}$, randomization test) and beta-band power was reduced (frequencies of reduction: 22-26 Hz, Monkey $\mathrm{B}, p<0.05,18-34 \mathrm{~Hz}$; Monkey $\mathrm{O}, p<0.05$, randomization test).

The gamma-frequency spectral modulations were consistent over recording sessions. In V1, all sessions showed gamma band increases with visual stimulation (range of percentage increase of gamma power from baseline in V1: 3 to $1868 \%$ increase, mean = $210 \%)$. The variability in gamma-band power was likely a result of the laminar position of the V1 electrode, which was variable over sessions (Maier et al., 2010; Buffalo et al., 2011; Xing et al., 2012 b). In the LGN, gamma power modulations were inconsistent over sessions (16 sessions showed weak decreases, 5 showed weak increases, most sessions showed $<10 \%$ modulation in either direction). Therefore, consistent stimulus-dependent gamma-frequency power increases were restricted to the cortex and not observed in the LGN.

To determine at which frequencies the LGN and V1 interact, we investigated oscillatory synchronization using the PPC measure (Vinck et al., 2010). The PPC spectrum between the LGN and cortex displayed a pronounced peak in the beta range $(28 \mathrm{~Hz}$ in Monkey B, $20 \mathrm{~Hz}$ in Monkey O; Fig. 3C,D). We next examined directed interactions using the PSI (Nolte et al., 2008). In both monkeys, we observed two prominent peaks in the PSI spectra, indicating feedforward interactions in the beta-frequency range (16-28 Hz in Monkey B, 14-26 Hz in Monkey O) and feedback interactions in the alpha-frequency range $(6-12 \mathrm{~Hz}$ in Monkey B, $8-12 \mathrm{~Hz}$ in Monkey O; Fig. 3E, F; $p<0.05$, randomization test). These findings indicate that gamma-band synchronization in V1 is likely produced via cortical mechanisms, because at the $\mathrm{V} 1$ gamma frequencies that were most strongly enhanced by visual 
stimulation $(44 \mathrm{~Hz}$ in Monkey B and 52 $\mathrm{Hz}$ in Monkey $\mathrm{O}$; Fig. $3 A, B$ ), directed interactions between the LGN and cortex were not significantly different from zero (Fig. $3 E, F$ ).

To rule out the possibility that gamma oscillations were present in the spiking activity of LGN neurons but not detectable in the LFP, we analyzed single-unit spiking data from two additional alert monkeys performing a similar fixation task while recorded neurons were excited with a drifting grating stimulus. Using $500 \mathrm{~ms}$ windows and multitaper spectral estimation with $8 \mathrm{~Hz}$ smoothing together with randomization testing, we tested whether the spiking activity of single neurons $(n=$ 209; 84 cells in Monkey C, 52 P cells and $32 \mathrm{M}$ cells); 125 cells in Monkey Bl, $58 \mathrm{P}$ cells and $67 \mathrm{M}$ cells) showed significant gamma-band power (relative to a spike shuffle control) that was also enhanced by visual stimulation. Furthermore, because Fourier analysis of spiking activity can result in frequency components that are related to bursting and refractory period behavior but not oscillations (Mureşan et al., 2008), we examined whether these cells had oscillatory side lobes in the autocorrelograms corresponding to the peak frequency in the gamma-band. In Monkey $\mathrm{C}$, no cells showed gamma oscillations according to these criteria, whereas in Monkey Bl, only two cells showed gamma oscillations (data not shown). These results are consistent with those from the LFP analyses (Figs. 1-3), indicating that LGN temporal dynamics are either very weakly or not at all oscillatory in the gamma-frequency band.

Last, we hypothesized that if gamma-band power increases in V1 are directly derived from LGN inputs, we should see a systematic relationship between LGN-V1 interactions, as measured with the PPC, and gamma power in V1. To test this, we computed the correlation between the LGN-V1 PPC in the $8-38 \mathrm{~Hz}$ range and power in $\mathrm{V} 1$ at each frequency from 2 to $100 \mathrm{~Hz}$, across recording sessions (Fig. $4 A$ ). The resulting correlation spectrum revealed that whereas LGN-V1 phase synchronization was positively correlated with low-frequency V1 power $(2-10 \mathrm{~Hz}$, permutation test for multiple comparisons, $r=0.71, p<0.05$; Fig. $4 B$ ), it was negatively correlated with gamma power in V1 $(26-48 \mathrm{~Hz}$, same test, $r=-0.70, p<0.05$; Fig. $4 C$ ), and not significantly correlated with high-gamma $(50-100 \mathrm{~Hz})$ power. These results suggest that V1 populations that are the most strongly driven by direct feedforward LGN inputs are the least gamma-modulated by visual stimulation. Together, these results support the notion that visually evoked gamma oscillations arise in the cortex.

\section{Discussion}

We made simultaneous recordings of LFPs from retinotopically corresponding regions of the LGN and V1 in alert macaque monkeys to investigate the presence, strength, and communication of rhythmic activity in the two structures. Whereas visual stimulation induced robust gamma-band oscillations in V1, it did not induce rhythmic gamma-band activity in the LGN. Therefore, communication between LGN and V1 in alert animals during visual stimulation is not dependent on subcortical-cortical gamma synchronization. Interestingly, however, subcorticalcortical synchronization was present between the LGN and V1 at lower-frequencies, peaking at 20 and $28 \mathrm{~Hz}$ and encompassing a broad range of frequencies from $\sim 8$ to $38 \mathrm{~Hz}$. Within this frequency range, analysis of directed influences revealed that the alpha-band (8-12 Hz) reflected feedback (V1 to LGN) influences, whereas the beta-band $(15-30 \mathrm{~Hz})$ reflected feedforward (LGN to V1) influences.

A correlation between the strength of LGN-V1 oscillatory synchronization with V1 gamma-band power across sessions revealed a negative correlation suggesting that V1 gamma oscillations are not directly inherited from feedforward LGN input. At the same time, V1 low-frequency $(2-10 \mathrm{~Hz})$ power was positively correlated with LGN-V1 interactions. Previous studies have shown that whereas supragranular V1 populations (above layer $4 \mathrm{C}$ ) express the strongest gamma power within V1, infragranular (L5/6) V1 populations express the strongest lowfrequency power (Xing et al., 2012b; Smith et al., 2013). Therefore, the most likely interpretation of the correlations between LGN-V1 oscillatory synchronization and V1 power is that sessions with relatively strong gamma-band power in V1 recorded activity from the supragranular layers, whereas sessions with relatively strong low-frequency power in V1 sampled neu- 
A

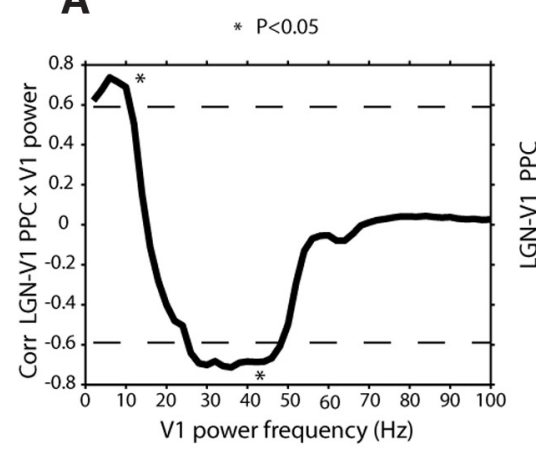

B

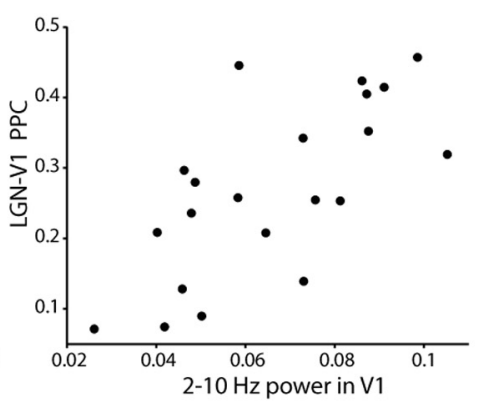

C

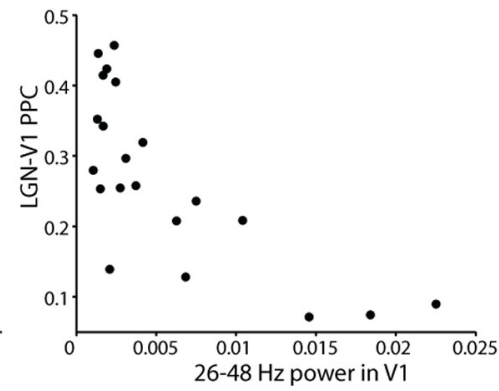

D

L1

V1

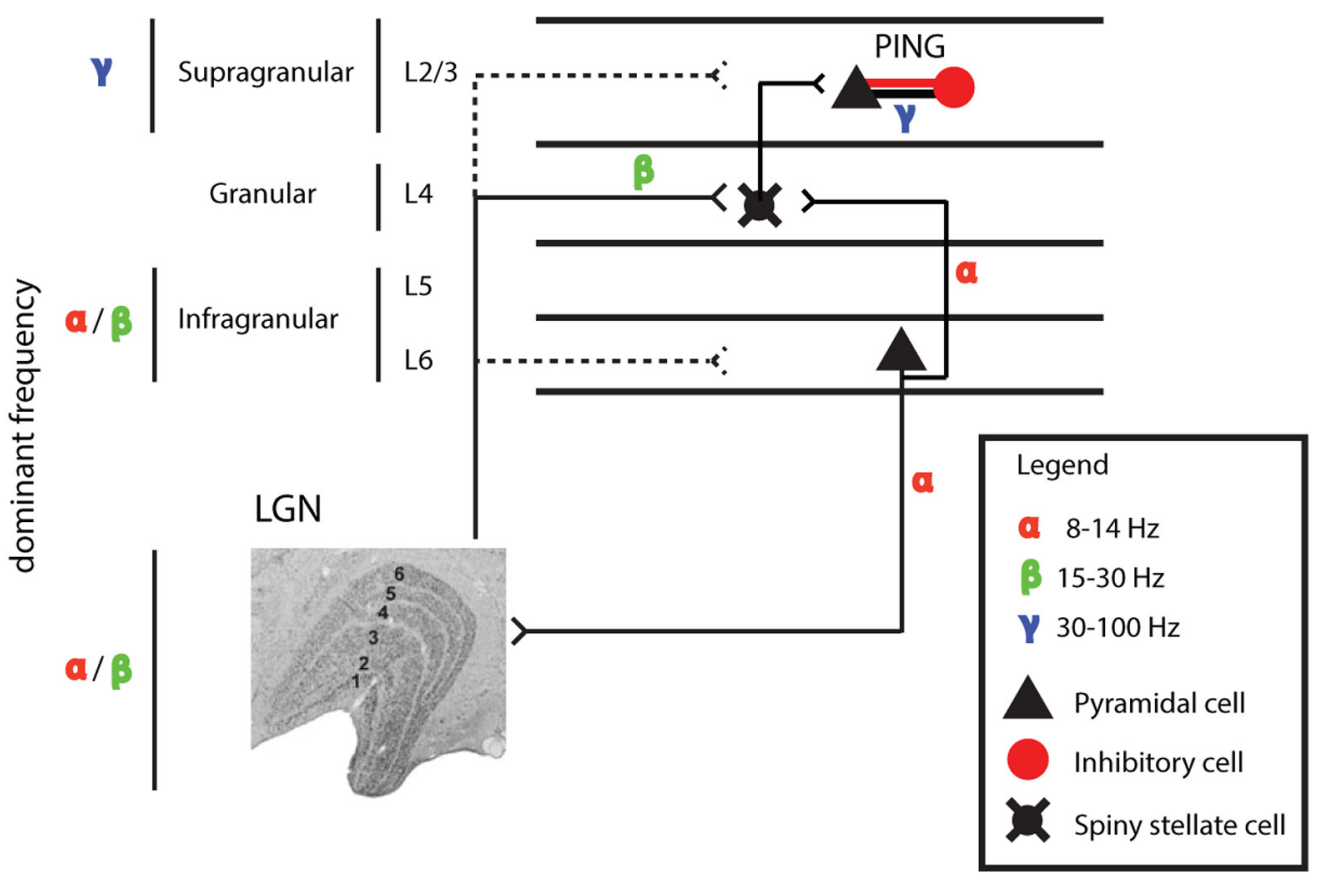

Figure 4. Session by session correlation of LGN-V1 PPC and V1 power, and schematic of LGN-V1 connectivity. $A$, Correlation spectrum between LGN-V1 PPC and V1 power. Dashed lines denote correlation values that are significant at $p<0.05$. B, C, Power by PPC scatter plots of the V1 frequencies $(\boldsymbol{B}, 2-10 \mathrm{~Hz} ; \boldsymbol{C}, 26-48 \mathrm{~Hz})$ that are significantly positively $(\boldsymbol{B})$ and negatively $(\boldsymbol{C})$ correlated with LGN-V1 PPC across sessions $(N=21)$. D, Schematic of functional and anatomical connections. The lines interconnecting areas or subpopulations indicate anatomical connections. Dotted lines indicate secondary (weaker) projections. Where functional data exist to indicate which frequency bands mediate the synaptic interactions (Maier et al., 2010; Buffalo et al., 2011; Xing et al., 2012b), the corresponding frequency is indicated.

ronal activity from the infragranular layers. This would mean that the V1 layers with strongest reciprocal anatomical connectivity to the LGN (granular and infragranular) are also the layers that synchronize to the LGN with oscillations in the subgamma range. Future studies with laminar resolution will be needed to confirm these predictions.

Previous reports have described robust gamma-frequency $(25-110 \mathrm{~Hz})$ oscillatory activity in the spike trains of neurons in the retina, LGN, and V1 (Neuenschwander and Singer, 1996; Castelo-Branco et al., 1998). Moreover, intracellular measurements indicate that cells in the retina and LGN are able to code visual information in spike times relative to the ongoing gamma oscillation (Koepsell et al., 2009). Therefore, it is important to consider why gamma-band activity is not evident in the activity of LGN neurons in the current study. One possibility is that the differences may be due to the species studied. Previous reports of gamma oscillations in the LGN come from studies using anesthetized cats. In the cat, recurrent connections between excitatory cells and inhibitory cells, which are thought to be necessary to establish pyramidal-interneuron network gamma (PING; Buzsáki and Wang, 2012), are present within the LGN (Bickford et al., 2008). However, such connectivity, to our knowledge, has not been found in the macaque LGN. Another possibility is the use of anesthesia. The current study was conducted using alert monkeys. To our knowledge, gamma-band oscillations in the LGN have not been reported in the alert state. Furthermore, recent studies indicate that anesthesia profoundly modulates gammaband synchronization in visual cortex compared with the alert state and may affect gamma in different ways depending on the anesthetic agent and its synaptic and network-level effects (Xing et al., 2012a; Saxena et al., 2013).

The results from the current study reveal synchronization between the LGN and V1 at alpha and beta frequencies, with the faster frequency (beta) involved in feedforward communication and the slower frequency (alpha) involved in feedback communication. A dissociation between higher and lower frequencies for 
transferring feedforward and feedback information, respectively, has been proposed as a general mechanism for corticocortical communication (Bastos et al., 2012). This segregation of putative feedforward and feedback processing through faster and slower frequencies has also been observed in corticostriatal (Litvak et al., 2012), and hippocampus to entorhinal cortex (Colgin et al., 2009) interactions, and therefore may be a generic mechanism across systems. Although speculative, this property of neuronal activity could provide a means for brain areas to segregate input and output activity (Bastos et al., 2012), and may play an important role in signaling top-down predictions and bottom-up prediction errors among different hierarchical processing levels (Friston, 2008).

Considering the results of the present study in combination with previous studies, we propose the following model of LGN-V1 dynamics (Fig. 4D). In this model, the LGN provides strong feedforward input with energy in the beta-frequency band to cells in layer $4 \mathrm{C}$ of $\mathrm{V} 1$. These cell populations provide strong, intrinsic "feedforward" connections to the superficial layers (Alonso and Martinez, 1998). Pyramidal cells and interneurons in the superficial layers, with reciprocal connections interconnecting them, could then form a minimal circuit capable of generating a gamma-frequency rhythm (a "PING" network; Buzsáki and Wang, 2012). Cells in the infragranular layers of V1, including corticogeniculate neurons in layer 6 , then synchronize their responses in a lower frequency band (Bollimunta et al., 2011; Buffalo et al., 2011; $<20 \mathrm{~Hz}$ ), leading to the observed directed corticogeniculate influences in the alpha range (Fig. $3 E, F$ ). Future studies with laminar resolution will be needed to confirm these predictions, and to discover which mechanisms mediate both the functional segregation and integration of oscillatory synchronization at different frequencies.

\section{References}

Alonso JM, Martinez LM (1998) Functional connectivity between simple cells and complex cells in cat striate cortex. Nat Neurosci 1:395-403. CrossRef Medline

Bastos AM, Usrey WM, Adams RA, Mangun GR, Fries P, Friston KJ (2012) Canonical microcircuits for predictive coding. Neuron 76:695-711. CrossRef Medline

Bickford ME, Wei H, Eisenback MA, Chomsung RD, Slusarczyk AS, Dankowsi AB (2008) Synaptic organization of thalamocortical axon collaterals in the perigeniculate nucleus and dorsal lateral geniculate nucleus. J Comp Neurol 508:264-285. CrossRef Medline

Bollimunta A, Mo J, Schroeder CE, Ding M (2011) Neuronal mechanisms and attentional modulation of corticothalamic alpha oscillations. J Neurosci 31:4935-4943. CrossRef Medline

Bosman CA, Schoffelen JM, Brunet N, Oostenveld R, Bastos AM, Womelsdorf T, Rubehn B, Stieglitz T, De Weerd P, Fries P (2012) Attentional stimulus selection through selective synchronization between monkey visual areas. Neuron 75:875-888. CrossRef Medline

Briggs F, Usrey WM (2009) Parallel processing in the corticogeniculate pathway of the macaque monkey. Neuron 62:135-146. CrossRef Medline

Briggs F, Mangun GR, Usrey WM (2013) Attention enhances synaptic efficacy and the signal-to-noise ratio in neural circuits. Nature 499:476-480. CrossRef Medline

Buffalo EA, Fries P, Landman R, Buschman TJ, Desimone R (2011) Laminar differences in gamma and alpha coherence in the ventral stream. Proc Natl Acad Sci U S A 108:11262-11267. CrossRef Medline

Buschman TJ, Miller EK (2007) Top-Down versus bottom-up control of attention in the prefrontal and posterior parietal cortices. Science 315: 1860-1862. CrossRef Medline

Buzsáki G, Wang XJ (2012) Mechanisms of gamma oscillations. Annu Rev Neurosci 35:203-225. CrossRef Medline

Castelo-Branco M, Neuenschwander S, Singer W (1998) Synchronization of visual responses between the cortex, lateral geniculate nucleus, and retina in the anesthetized cat. J Neurosci 18:6395-6410. Medline

Colgin LL, Denninger T, Fyhn M, Hafting T, Bonnevie T, Jensen O, Moser MB, Moser EI (2009) Frequency of gamma oscillations routes flow of information in the hippocampus. Nature 462:353-357. CrossRef Medline

Fries P (2005) A mechanism for cognitive dynamics: neuronal communication through neuronal coherence. Trends Cogn Sci 9:474-480. CrossRef Medline

Friston K (2008) Hierarchical models in the brain. PLoS Comput Biol 4:e1000211. CrossRef Medline

Gregoriou GG, Gotts SJ, Zhou H, Desimone R (2009) High-frequency, long-range coupling between prefrontal and visual cortex during attention. Science 324:1207-1210. CrossRef Medline

Koepsell K, Wang X, Vaingankar V, Wei Y, Wang Q, Rathbun DL, Usrey WM, Hirsch JA, Sommer FT (2009) Retinal oscillations carry visual information to cortex. Front Syst Neurosci 3:4. CrossRef Medline

Litvak V, Eusebio A, Jha A, Oostenveld R, Barnes G, Foltynie T, Limousin P, Zrinzo L, Hariz MI, Friston K, Brown P (2012) Movement-related changes in local and long-range synchronization in Parkinson's disease revealed by simultaneous magnetoencephalography and intracranial recordings. J Neurosci 32:10541-10553. CrossRef Medline

Maier A, Adams GK, Aura C, Leopold DA (2010) Distinct superficial and deep laminar domains of activity in the visual cortex during rest and stimulation. Front Syst Neurosci 4:31. CrossRef Medline

Mitra PP, Pesaran B (1999) Analysis of dynamic brain imaging data. Biophys J 76:691-708. CrossRef Medline

Mureşan RC, Jurjuţ OF, Moca VV, Singer W, Nikolić D (2008) The oscillation score: an efficient method for estimating oscillation strength in neuronal activity. J Neurophysiol 99:1333-1353. CrossRef Medline

Neuenschwander S, Singer W (1996) Long-range synchronization of oscillatory light responses in the cat retina and lateral geniculate nucleus. Nature 379:728-732. CrossRef Medline

Nolte G, Ziehe A, Nikulin VV, Schlögl A, Krämer N, Brismar T, Müller KR (2008) Robustly estimating the flow direction of information in complex physical systems. Phys Rev Lett 100:234101. CrossRef Medline

Oostenveld R, Fries P, Maris E, Schoffelen JM (2011) FieldTrip: open source software for advanced analysis of MEG, EEG, and invasive electrophysiological data. Comput Intell Neurosci 2011:156869. CrossRef Medline

Roberts MJ, Lowet E, Brunet NM, Ter Wal M, Tiesinga P, Fries P, De Weerd P (2013) Robust gamma coherence between macaque V1 and V2 by dynamic frequency matching. Neuron 78:523-536. CrossRef Medline

Saxena N, Muthukumaraswamy SD, Diukova A, Singh K, Hall J, Wise R (2013) Enhanced stimulus-induced gamma activity in humans during propofol-induced sedation. PloS One 8:e57685. CrossRef Medline

Smith MA, Jia X, Zandvakili A, Kohn A (2013) Laminar dependence of neuronal correlations in visual cortex. J Neurophysiol 109:940-947. CrossRef Medline

Vijayan S, Ching S, Purdon PL, Brown EN, Kopell NJ (2013) Thalamocortical mechanisms for the anteriorization of alpha rhythms during propofolinduced unconsciousness. J Neurosci 33:11070-11075. CrossRef Medline

Vinck M, van Wingerden M, Womelsdorf T, Fries P, Pennartz CM (2010) The pairwise phase consistency: a bias-free measure of rhythmic neuronal synchronization. Neuroimage 51:112-122. CrossRef Medline

Xing D, Shen Y, Burns S, Yeh CI, Shapley R, Li W (2012a) Stochastic generation of gamma-band activity in primary visual cortex of awake and anesthetized monkeys. J Neurosci 32:13873-13880a. CrossRef Medline

Xing D, Yeh CI, Burns S, Shapley RM (2012b) Laminar analysis of visually evoked activity in the primary visual cortex. Proc Natl Acad Sci U S A 109:13871-13876. CrossRef Medline 